\section{Studies on mutation breeding in mandarin variety Kinnow}

\section{Harinder Singh Rattanpal*, Gurteg Singh and Monika Gupta}

Department of Fruit Science, Punjab Agricultural University, Ludhiana 141004 , India

The present study was undertaken to generate variability in Kinnow (Citrus nobilis Lour $\times C$. deliciosa Tenora) using gamma radiation. Four hundred buds of Kinnow were irradiated at $30 \mathrm{~Gy}$ and budded on rough lemon rootstock in February 2007. The resulting $188 \mathrm{MV}_{1}$ plants were planted in the field in October 2009 and observations in the present trial were recorded from 2015 to 2018 . The number of bold seed per fruit ranged from 0.4 to 30.8 in the population raised from irradiated buds. Out of all the branches, $6.4 \%$ had bold seed number less than 10.3 per fruit, $39.4 \%$ had bold seed number from 10.3 to 20.3 per fruit and $54.7 \%$ plants had bold seed number more than 20.3 per fruit. The average fruit weight in treated population ranged from 94.3 to $253.2 \mathrm{~g}$, with $\mathbf{4 6 . 8 \%}$ bearing small sized fruits, $44.3 \%$ bearing medium sized fruits and $8.9 \%$ bearing large sized fruits. High variability was also observed for plant height, plant spread, pollen viability and total soluble solids. Among the $188 \mathrm{MV}_{1}$ plants, eleven Kinnow mutants (seven solid mutant trees and four mutant branches) having average seed number less than eight were identified, showing distinct desirable characters and were compared in detail with parent variety Kinnow. One of the low seeded mutants was released as a new variety named 'PAU Kinnow-1' for cultivation in Indian Punjab.

Keywords: Flowering characters, fruit weight, pollen viability, seedless.

THE Kinnow mandarin (Citrus nobilis Lour $\times$ C. deliciosa Tenora) has emerged as a predominant citrus fruit in Punjab and is grown on 51.6 thousand hectare with an annual production of 12.1 lakh metric tonnes (ref. 1). Kinnow trees are precocious, have wide adaptability and are heavy yielders of good quality fruits with abundant juice. Fruits have high juice content with well blended taste. However, this cultivar has a problem of high seed number and this problem was the biggest hindrance in its adoption as a table fruit. Seed number also adds bitterness to the juice after squeezing of juice. On the other hand seedless cultivars are preferred by the trade. Citrus are highly heterozygous, polygenic plants with a long juvenile period, whereby conventional breeding is laborious, timeconsuming and expensive.

\footnotetext{
*For correspondence. (e-mail: hsrattanpal@pau.edu)
}

Inducing mutations by gamma-rays has been effectively used in several species of Citrus. Irradiation of budwood with gamma-rays can produce higher frequencies of mutation, leading to the creation of new variants compared to the parent variety. Gamma-irradiation mutagenesis of budwood has been the most commonly used method by citrus breeders worldwide to obtain seedless clones from commercial seeded varieties ${ }^{2,3}$ of orange, mandarin, grapefruit and lemon ${ }^{4,5}$. An alternative method to obtain seedless citrus fruit is through breeding of triploid trees; however, this method presents several drawbacks, such as prolonged juvenility, segregation of characters on crossing and hybrids with long thorns ${ }^{6}$. Unlike other mutagenesis methods, gamma irradiation at the doses used to induce seedlessness often results in induction of numerous recessive chromosomal aberrations. Therefore, it is very likely that it impacts the expression of many genes and thereby in some cases, impairs fruit quality ${ }^{2,3}$.

Despite the vast efforts invested in the development of seedless mandarin varieties, not much is yet known regarding the effects of gamma-irradiation mutagenesis and/or lack of seeds on mandarin fruit quality. In studies, conducted in Spain, Bermejo et al. ${ }^{7,8}$ compared seed number, pollen viability and fruit quality traits of bud irradiated 'Moncada' and 'Murcott' mandarin clones with standard trees. They observed that some of irradiationgenerated clones had reduced pollen germination rates and thereby had fewer seeds. However, irradiation also resulted in alterations in fruit weight, size and nutritional quality, though some clones exhibited no changes in fruit-quality traits when compared to fruits harvested from standard trees. Therefore, the present study was planned to assess the variability induced in Kinnow population with gamma irradiation and to study fruit quality characters of mutants having average seed number less than eight at phenotypic level.

The study was undertaken in the New Research Farm of Punjab Agricultural University, Ludhiana. The gammairradiated clones were obtained by grafting buds that had been exposed to $30 \mathrm{~Gy}$ of ${ }^{60} \mathrm{Co}$ gamma irradiation onto 'rough lemon' rootstock. The buds were exposed to gamma rays in a gamma chamber emitting $11.2 \mathrm{~Gy} / \mathrm{min}$. A dose of $50 \%$ lethality of gamma rays $\left(\mathrm{LD}_{50}\right.$ dose) was standardized and it was observed to be 30 Gy for Kinnow buds. Four hundred Kinnow buds were irradiated in February 2007 and the resulting 188 first mutant generation $\left(\mathrm{MV}_{1}\right)$ plants were planted in the field in October 2009 at a spacing of $6 \times 3 \mathrm{~m}$, which were headed back to six buds after four months of growth. Two to four branches were retained on each plant. Fruit data for individual branch was recorded for the fruiting years $2015-16$ to $2017-18$. Tree height, tree spread and scion diameter were recorded for all the main branches of $188 \mathrm{MV}_{1}$ trees and five trees of parent variety Kinnow. Mutant-1, Mutant-2, Mutant-3, Mutant-4, Mutant-5, Mutant-6 and Mutant-11 were solid mutants and seed number per fruit and percentage of 
Table 1. Characterization of Kinnow mutants with respect to different characters

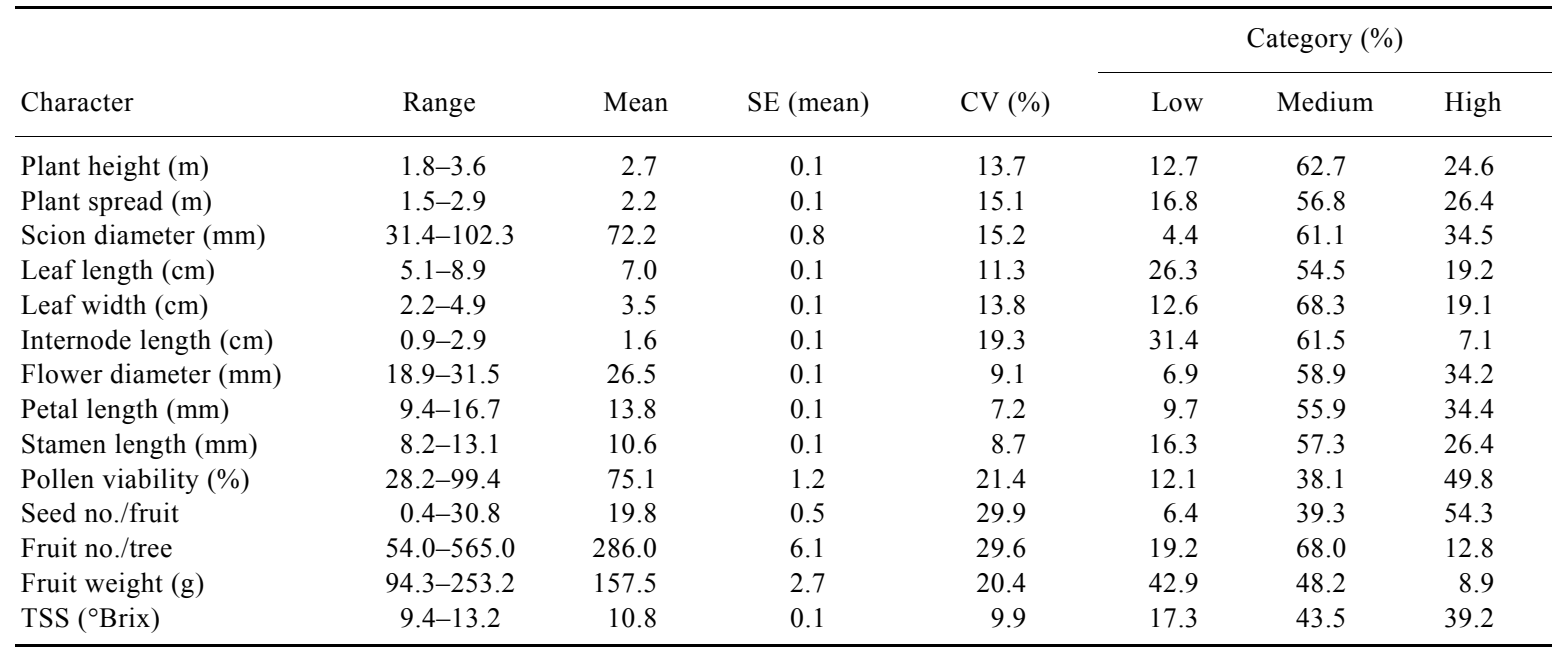

pollen viability per tree were uniformly same on the whole tree; however, the remaining four mutants were individual branches identified based on having less than eight bold seed per fruit and reduced pollen viability.

Data on different vegetative characters, viz. tree height, tree spread, scion diameter, leaf length and width were recorded during January. Pollen viability was determined with acetocarmine colorimetric test. The pollen grains from mature anthers were stained with $2 \%$ acetocarmine and observations were recorded under photonic microscope. The pollen viability was scored according to staining level (pollen with bold red colour as viable and colourless as non-viable). The percentage of pollen viability was determined as the ratio of the number of fertile grains to the total grain number. Flower diameter, petal length and stamen length were measured with Vernier's Callipers.

The Kinnow fruits were harvested in mid-January and a representative sample of 20 fruits was collected from each marked branch of a tree for analysis, and data on fruit weight, total soluble solids and acidity were recorded. Seeds were extracted and divided into two categories, i.e. bold and rudimentary depending upon their size; small shrivelled seeds were taken as rudimentary. Seed weight, length and diameter were recorded after drying the seed for $48 \mathrm{~h}$ at room temperature. All the fruits produced on a tree were counted to get the fruit number per tree. Tree height, tree spread, scion diameter and fruit number/tree were recorded for the whole tree. All other characters were recorded for individual branches.

The data were subjected to statistical analysis for standard error (SE) and coefficient of variation (CV). The range represented the minimum and maximum values recorded for each trait. The range for each character was divided into three equal parts which were designated as low, medium and high. The number of mutants falling in each category was calculated and expressed as a percen- tage of the total population, i.e. the total number of plants or branches.

In all the Kinnow mutant branches under evaluation, the range of seed number per fruit was recorded from 0.4-30.8 (Table 1). The mean number of seeds per fruit in the entire mutant population was 19.8. High variability expressed in terms of coefficient of variation (29.9\%) was recorded for seed number, which indicates that selection can be applied for seed number to isolate more promising lines. Though high variability was observed in Kinnow $\mathrm{MV}_{1}$ population, only $6.4 \%$ branches had low bold seed number/fruit and most of the branches (54.7\%) produced high bold seed number/fruit. Similar results were obtained in previous studies on mutation breeding of mandarins $s^{7,8}$. Lower seed number per fruit in different citrus species through mutation breeding was also obtained by Hearn ${ }^{9}$ and Spiegel Roy ${ }^{10}$. Seedless mandarins have been selected from bud variations of seedy cultivars and origin of bud variation was a change in the genetic constitution, including changes in chromosome number per cell, chromosome structure aberration and gene mutations ${ }^{11}$.

The number of fruits per tree ranged from 54 to 565 and mean number of fruits per tree was 286 (Table 1). High coefficient of variation of $29.6 \%$ was recorded in mutant population. Most of the trees (68\%) produced medium number of fruits; proportion of trees yielding high or low number of fruits per tree was low. Likewise, yield of 'Tayl' derived through spontaneous mutation of 'CK1' was found to be high and stable ${ }^{12}$.

Average fruit weight of each branch of the tree was recorded individually and it ranged from 94.3 to $253.2 \mathrm{~g}$ per fruit, with mean fruit weight of $157.5 \mathrm{~g}$ (Table 1). Variability of $20.4 \%$ was recorded in the mutant population. Most of the branches (48.2\%) produced medium sized fruits, followed by branches producing small sized fruits $(42.9 \%)$. In a similar study, Li et al. ${ }^{12}$ observed that 
RESEARCH COMMUNICATIONS

Table 2. Performance of Kinnow mutants for bold and rudimentary seed number per fruit

\begin{tabular}{|c|c|c|c|c|c|c|c|c|}
\hline \multirow[b]{2}{*}{ Mutant/character } & \multicolumn{4}{|c|}{ Bold seed number/fruit } & \multicolumn{4}{|c|}{ Rudimentary seed number/fruit } \\
\hline & $2015-16$ & $2016-17$ & $2017-18$ & Mean & $2015-16$ & $2016-17$ & $2017-18$ & Mean \\
\hline Mutant-1 & 4.12 & 3.22 & 3.73 & 3.69 & 8.63 & 8.24 & 9.18 & 8.68 \\
\hline Mutant-2 & 5.17 & 4.88 & 3.82 & 4.62 & 16.40 & 16.11 & 15.27 & 15.93 \\
\hline Mutant-3 & 6.59 & 7.96 & 6.72 & 7.09 & 13.31 & 11.74 & 10.33 & 11.79 \\
\hline Mutant-4 & 7.08 & 7.72 & 8.04 & 7.61 & 6.32 & 7.22 & 5.44 & 6.33 \\
\hline Mutant-5 & 1.62 & 0.38 & 1.41 & 1.14 & 3.22 & 4.45 & 4.56 & 4.08 \\
\hline Mutant-6 & 4.20 & 3.62 & 5.68 & 4.50 & 6.50 & 2.90 & 3.93 & 4.44 \\
\hline Mutant-7 & 6.41 & 7.67 & 7.90 & 7.33 & 11.33 & 12.56 & 13.21 & 12.37 \\
\hline Mutant-8 & 6.12 & 7.71 & 4.20 & 6.01 & 5.44 & 7.14 & 6.51 & 6.36 \\
\hline Mutant-9 & 8.21 & 3.00 & 6.11 & 5.77 & 15.28 & 16.51 & 14.78 & 15.52 \\
\hline Mutant-10 & 5.85 & 6.50 & 6.61 & 6.32 & 8.30 & 6.17 & 4.25 & 6.24 \\
\hline Mutant-11 & 6.66 & 8.25 & 7.37 & 7.43 & 5.92 & 5.50 & 5.67 & 5.70 \\
\hline Kinnow & 21.27 & 20.12 & 22.42 & 21.27 & 20.89 & 21.69 & 25.43 & 22.67 \\
\hline
\end{tabular}

Table 3. Performance of Kinnow mutants for average fruit weight

\begin{tabular}{lcccc}
\hline & \multicolumn{4}{c}{ Fruit weight (g) } \\
\cline { 2 - 5 } Mutant/character & $2015-16$ & $2016-17$ & $2017-18$ & Mean \\
\hline Mutant-1 & 167.8 & 182.2 & 175.2 & 175.1 \\
Mutant-2 & 152.3 & 125.1 & 137.5 & 138.3 \\
Mutant-3 & 178.6 & 177.3 & 154.6 & 170.2 \\
Mutant-4 & 190.3 & 209.2 & 191.2 & 196.9 \\
Mutant-5 & 86.4 & 94.3 & 76.33 & 85.7 \\
Mutant-6 & 118.6 & 108.4 & 127.5 & 118.2 \\
Mutant-7 & 188.4 & 189.2 & 183.5 & 187.0 \\
Mutant-8 & 162.1 & 173.4 & 177.5 & 171.0 \\
Mutant-9 & 164.5 & 176.4 & 169.5 & 170.1 \\
Mutant-10 & 168.8 & 168.1 & 174.4 & 170.4 \\
Mutant-11 & 169.3 & 173.2 & 178.6 & 173.7 \\
Kinnow & 171.6 & 172.9 & 182.5 & 175.7 \\
\hline
\end{tabular}

fruits of mutant 'Tay1' were larger, which was derived through spontaneous mutation of 'CK1'. Goldenberg et $a l .{ }^{13}$ observed that average fruit weights of seven of the eight low-seeded irradiated mandarin varieties were significantly lower than those of varieties from unirradiated trees.

Total soluble solids in mutated population ranged from 9.4 to $13.2^{\circ} \mathrm{Brix}$, and the mean TSS was $11.5^{\circ} \mathrm{Brix}$ (Table 1). The coefficient of variation for TSS was only $5.9 \%$ indicating low probability of selection for this trait. Most of the population produced fruits with medium to high TSS. Similar variation for TSS was observed in seedless mutants obtained through irradiation by Tang et al. ${ }^{14}$.

Diameter of flowers differed in mutant population. The flower diameter ranged from 18.9 to $31.5 \mathrm{~mm}$, with mean of $26.5 \mathrm{~mm}$ and coefficient of variation was $6.9 \%$ (Table 1). Most flowers (58.9\%) had medium diameter and $34.4 \%$ flowers were in the large diameter category. A similar trend was observed for petal length, which ranged from 9.4 to $16.7 \mathrm{~mm}$, with mean petal length of $13.8 \mathrm{~mm}$. Coefficient of variation for this character was $7.2 \%$. Low coefficient of variation $(8.7 \%)$ was also recorded for stamen length, most of the flowers in mutant population had medium stamen length.

Pollen viability of the entire population was estimated using acetocarmine staining and it ranged from $28.2 \%$ to $99.4 \%$ (Table 1). The mean pollen viability of the entire population was $75.1 \%$ with coefficient of variation of $21.4 \%$. Most of the flowers $(49.8 \%)$ had high pollen viability and $39.1 \%$ flowers were in medium range. Male sterility in citrus is generally referred to as pollen abortion that plays a key role in creating seedlessness in the fruit. It can be induced by degeneration of pollen grains, anther and microsporocyte, chromosomal rearrangements that cause abnormal meiosis in microsporocytes, as well as abnormal climate ${ }^{12}$.

Plant height in mutant population ranged from 1.8 to $3.6 \mathrm{~m}$ (Table 1). The mean plant height of the entire population was $2.7 \mathrm{~m}$ and most of the plants (62.7\%) had medium height. The coefficient of variation for plant height was $13.7 \%$ and plant spread was $15.1 \%$. Spread of plants varied from 1.5 to $2.9 \mathrm{~m}$ with average of $2.2 \mathrm{~m}$. Like plant height, most plants were in the medium category for spread. Scion diameter ranged from 31.4 to $102.3 \mathrm{~mm}$. The average trunk diameter was $72.2 \mathrm{~mm}$ and coefficient of variation for this character was of medium level at $15.2 \%$. High coefficient of variation (19.3\%) was observed for internode length, though very few branches (7.1\%) had high internode length.

In the entire mutant population, leaf length ranged from 5.1 to $8.9 \mathrm{~cm}$, with average length of $7.0 \mathrm{~cm}$ (Table 1). The coefficient of variation for this character was $11.3 \%$. Most mutants have leaf size in the medium range. Change in leaf characters as a result of gamma ray induced mutations was also observed by Kukimura et $a l .{ }^{14}$, who recorded mutations with elongated leaves and found this trait to be sexually transmitted.

Seven solid mutants were identified from the population described above based on bold seed number per fruit. Consistently low bold seed number was observed in 


\section{RESEARCH COMMUNICATIONS}

Table 4. Performance of Kinnow mutants for TSS and acidity of fruit juice

\begin{tabular}{|c|c|c|c|c|c|c|c|c|}
\hline \multirow[b]{2}{*}{ Mutant/character } & \multicolumn{4}{|c|}{ TSS ( ${ }^{\circ}$ Brix $)$} & \multicolumn{4}{|c|}{ Acidity (\%) } \\
\hline & $2015-16$ & $2016-17$ & $2017-18$ & Mean & $2015-16$ & $2016-17$ & $2017-18$ & Mean \\
\hline Mutant-2 & 9.8 & 10.2 & 10.0 & 10.0 & 0.85 & 0.82 & 0.83 & 0.83 \\
\hline Mutant-3 & 10.3 & 10.4 & 9.8 & 10.2 & 0.79 & 0.81 & 0.81 & 0.80 \\
\hline Mutant-6 & 10.1 & 9.9 & 9.7 & 9.9 & 0.86 & 0.85 & 0.85 & 0.85 \\
\hline Mutant-7 & 10.3 & 10.1 & 10.5 & 10.3 & 0.79 & 0.77 & 0.78 & 0.78 \\
\hline Mutant-8 & 10.3 & 10.2 & 10.4 & 10.3 & 0.79 & 0.81 & 0.82 & 0.81 \\
\hline Mutant-9 & 10.4 & 10.2 & 10.3 & 10.3 & 0.841 & 0.83 & 0.83 & 0.83 \\
\hline Mutant-10 & 10.3 & 10.5 & 10.4 & 10.4 & 0.83 & 0.8 & 0.81 & 0.81 \\
\hline
\end{tabular}

(a)

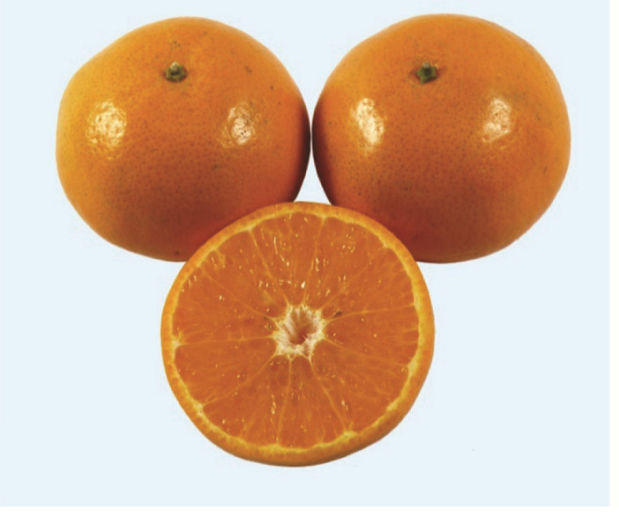

(b)

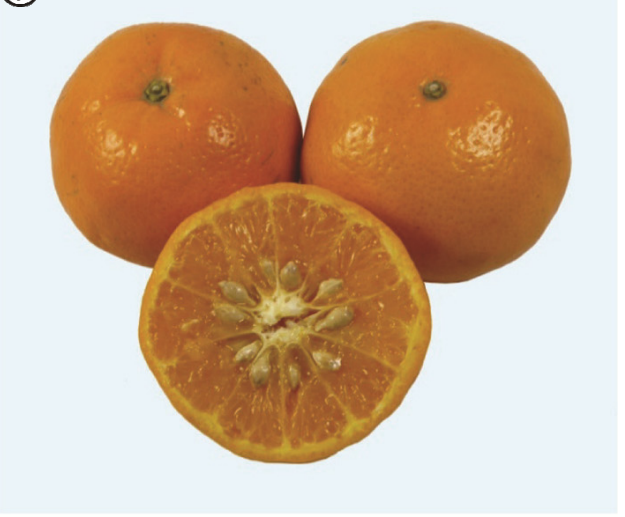

Figure 1. $\boldsymbol{a}$, Mutant-1 having low seed content was released as new variety named 'PAU Kinnow-1', b, Parent variety 'Kinnow'.

mutant-5 during all the years followed by mutant- 1 . The highest bold seed number per fruit was recorded in parent variety Kinnow (Table 2). Mutant-1 was released as a seedless mandarin variety named 'PAU Kinnow-1' for general cultivation in Punjab, India (Figure 1). Mutant-5 could not be released due to small sized fruits (Table 3) and low TSS (Table 4); average fruit weight in mutant-5 was less than half that of mutant-1. Average number of rudimentary seeds per fruit was the lowest in mutant-5 followed by mutant- 6 , mutant- 10 and mutant- 4 in ascending order. Like this study, a superior seedless mutant of 'Jing' orange 'Xifeng No. 1' was selected by Jiang et $a l .{ }^{15}$.

All the mutants did not differ much for fruit biochemical quality parameters (Table 4). Consistently low TSS was observed in mutant-5 closely followed by mutant-6. The lowest mean acidity was recorded in mutant- 4 followed by mutant- 6 . Similarly, spontaneous mutant named 'Autumn Gold Late Navel' was identified from 'Washington Navel' population by Pollock ${ }^{16}$.

The fruits of mutant-5 were consistently smaller in size compared to parent variety Kinnow and all other selected mutants (Table 3). Mutant-5 also had the lowest seed number per fruit. The fruits of mutant- 4 and mutant- 7 were larger in size. In mandarin cultivars irradiated with gamma rays, Goldenberg et al. ${ }^{13}$ observed that average fruit weight of seven of the eight low-seeded irradiated mandarin varieties was significantly lower than those of unirradiated trees.

Huge variability was observed in the mutant population for many traits of commercial significance. In the present trial, selection was focused on low seed number. As a result, seven solid mutants and four branches bearing fruits having less than eight seeds per fruit were identified. One of these mutants, mutant-1 was released as new variety named 'PAU Kinnow-1' for general cultivation in Punjab, India.

1. Anon., Statement showing the district wise area, average yield and production of various fruit crops for the year 2015-16 in the state of Punjab. Directorate of Horticulture, Government of Punjab, Chandigarh, 2018.

2. Gidoni, D. and Carmi, N., Mutagenesis for seedlessness in citrus. Isr. J. Plant Sci., 2007, 55, 133-135. 
3. Vardi, A., Levin, I. and Carmi, N., Induction of seedlessness in citrus: from classical techniques to emerging biotechnological approaches. J. Am. Soc. Hortic. Sci., 2008, 133, 117-126.

4. Roose, M. L. and Williams, T. E., Mutation breeding in citrus. In Citrus Genetics, Breeding and Biotechnology (ed. Khan, I. A.), CAB International, Wallingford, 2007, pp. 345-352.

5. Spiegel Roy, P., Vardi, A. and Elhanati, A., Seedless induced mutant in highly seeded lemon Citrus limon. Mutat. Breed. News, 1985, 26, 1-2

6. Aleza, P., Juarez, J., Hernandez, M., Ollitrault, P. and Navarro, L., Implementation of extensive citrus triploid breeding programs based on $4 \mathrm{x} \times 2 \mathrm{x}$ sexual hybridisations. Tree Genet. Genomes, 2012, 8, 1293-1306.

7. Bermejo, A., Pardo, J. and Cano, A., Influence of gamma irradiation on seedless citrus production: pollen germination and fruit quality. Food Nutr. Sci., 2011, 2, 169-180.

8. Bermejo, A., Pardo, J. and Cano, A., Murcott seedless: influence of gamma irradiation on citrus production and fruit quality. Span. J. Agric. Res., 2012, 10, 768-777.

9. Hearn, C. J., Development of seedless orange and grapefruit cultivars through seed irradiation. J. Am. Soc. Hortic. Sci., 1984, 109, 270-273.

10. Spiegel Roy, P., Economic and agricultural impact of mutation breeding in fruit trees. Mutat. Breed. Rev. Vienna, 1990, 5, 215235 .
11. Shen, D. X., Fruit Breeding, Chinese Agricultural Press, Beijing, 1997, 2nd edn.

12. Li, Z. Y. et al., A new seedless ponkan mutant - 'Tay1' ponkan. J. South China Agric. Univ., 1997, 18, 3.

13. Goldenberg, L., Yaniv Yossi, Porat Ron and Goldenberg, N. C., Effects of gamma-irradiation mutagenesis for induction of seedlessness, on the quality of mandarin fruit. Food Nutr. Sci., 2014, 5, 943-952.

14. Kukimura, H. et al., Genetical, cytological and physiological studies on the induced mutants with special regard to effective methods for obtaining useful mutants in perennial woody plant. II. Improvement of vegetatively propagated plants and tree crops through induced mutations. In Proceedings of the Second Research Coordination Meeting held at Wageningen, The Netherlands, 1976.

15. Jiang, J. G., Li, D. G., Fu, D. M., Jiang, J. G., Li, D. G., Fu, D. M. and Zhu, D. W., A superior seedless mutant clone of 'Jing' orange 'Xifeng No. 1'. Acta Hortic., 1995, 403, 96.

16. Pollock, J., Variety: 'Autumn Gold Late Navel'. Application no. 89/004. Plant Vars J., 1995, 8, 25-27.

Received 6 March 2018; revised accepted 13 November 2018

doi: $10.18520 / \mathrm{cs} /$ v116/i3/483-487 